\title{
HUSSERL Y EL ANTIRREALISMO (¿O REALISMO?) CIENTÍFICO
}

\author{
Juan Carlos Aguirre-García* \\ jcaguirre@unicauca.edu.co
}

RESUMO $O$ presente artigo se propõe a confrontar uma leitura antirrealista da obra do fenomenólogo Edmund Husserl e a sugerir que ela pode ser interpretada mais adequadamente como marco do realismo cientifico. Para tanto, primeiramente, será exposto um dos mais recentes e elaborados projetos nos quais Husserl se relaciona ao Empirismo Construtivo de Van Fraassen. Posteriormente, serão assinaladas as dificuldades encontradas para interpretar algumas teses de Husserl desde o marco antirrealista, enfocando, principalmente, a discussão sobre os inobserváveis, mostrando como a sua existência não é uma tese indiferente à fenomenologia husserliana. Por fim, será indicado como Husserl poderia estar mais próximo do marco realista.

Palavras-chave Antirrealismo, evidência, inobserváveis, instrumentos, realismo.

ABSTRACT In this paper I confront an anti-realistic approach to Edmund Husserl's Phenomenological work and I suggest that this work could most suitably constitute into the Scientific Realistic framework. In order to

* Profesor Asociado, Departamento de Filosofía, Universidad del Cauca (Colombia). Este artículo es producto del proyecto: "Las retóricas del construccionismo epistemológico: una aproximación a las concepciones de ciencia, realidad y racionalidad de los investigadores sociales de la Universidad del Cauca" (VRI-3376), adelantado al interior del grupo de investigación Fenomenología y Ciencia, y financiado por la Vicerrectoría de Investigaciones de la Universidad del Cauca - Colombia. El autor da expreso crédito y mención a la Universidad del Cauca por la financiación de la investigación. Igualmente, agradece la evaluación del árbitro anónimo, pues sus sugerencias permitieron reestructurar radicalmente el artículo. Artigo recebido em 03/08/2012 e aprovado em 20/05/2013.

KRITERION, Belo Horizonte, $n^{\circ}$ 129, Jun./2014, p. 287-308 
this, I will first show one of most recent and elaborate claims which relates Husserl with Van Fraassen's Constructive Empiricism. Later, I will point out some existing difficulties to interpret the Husserlian thesis using the antirealist framework. I will specially focus on the discussion about unobservable entities, arguing how the existence of the unobservable is not an alien thesis to the Phenomenology of Husserl. Finally, I will point out how Husserl is closer to the Realistic framework.

Keywords Anti-Realism, evidence, unobservable, instruments, Realism.

\section{Introducción}

Parece extraño considerar las tesis de Edmundo Husserl en el contexto del debate contemporáneo entre realismo/antirrealismo científico, pues tales tesis pertenecerían exclusivamente a la filosofía de la ciencia, disciplina tradicionalmente vista como opuesta o lejana a la fenomenología. Ciertamente, ambas disciplinas han estado navegando por océanos incomunicados casi que durante toda su historia, considerada esta desde el siglo veinte. Lo realmente extraño, sin embargo, es que no haya suficiente diálogo entre ellas, toda vez que la fenomenología, desde sus orígenes, se presentó como una teoría de la ciencia. Justamente, a comienzos de la década del setenta del siglo anterior, el fenomenólogo Aaron Gurwitsch declaraba que "en las décadas recientes la teoría de la ciencia no ha recibido suficiente atención en la literatura fenomenológica" (Gurwitsch, 1974, p. 31), planteando con bastante optimismo que "parece que ha llegado el momento para que la fenomenología reclame posesión del campo que tuvo como punto de partida los escritos iniciales de Husserl" (ibidem, p. 32). Pese a esto, los trabajos que relacionan la fenomenología con la filosofía de la ciencia han sido escasos.

No obstante, el profesor Harald Wiltsche ha publicado recientemente un artículo titulado: "What is Wrong with Husserl's Scientific Anti-Realism?", en el que se evidencia cómo la filosofía de Husserl puede entrar en diálogo directo con asuntos propios de la filosofía de la ciencia contemporánea, específicamente con el debate entre realismo y antirrealismo científico. De cierta manera, Wiltsche reactualiza la inquietud que varias décadas atrás hacía Gurwitsch, preguntándose: “¿cómo debería un fenomenólogo de hoy, que quiere ser fiel al programa inicial de Husserl, reaccionar ante el actual debate sobre el realismo científico?" (Wiltsche, 2012, p. 107). La pregunta como tal no es absolutamente original, la misma se ha venido haciendo poco tiempo 
después de que Gurwitsch enunciara el desafío. ${ }^{1}$ Lo original consiste en el tratamiento que le da a la misma y su apuesta por relacionar la fenomenología de Husserl con el antirrealismo científico del reconocido filósofo de la ciencia Bas C. Van Fraassen, en especial con su propuesta del Empirismo Constructivo. Pese a la originalidad del tratamiento y a la importante contribución del documento para la consolidación de una fenomenología de la ciencia o una filosofía de la ciencia con aportes de la fenomenología, la lectura que hace Wiltsche y su intento por relacionar a Husserl con el programa del Empirismo Constructivo, no están exentos de dificultades. El propósito del presente artículo es, precisamente, controvertir algunas interpretaciones que hace Wiltsche del trabajo de Husserl. El interés no es solo entablar una discusión con Wiltsche, sino mostrar otra alternativa a tal interpretación, esta vez más cercana al Realismo científico. El presente texto, entonces, aspira a servir de balance a la hora de identificar la postura de Husserl con respecto a la ciencia, con una clara insinuación de que su perspectiva es más bien realista. No hay pretensión de agotar el asunto, pues como el fenomenólogo Drummond (1988) alguna vez lo afirmó: "[estos] debates son complicados no solo por las dificultades de los textos de Husserl, sino por el hecho de que los participantes frecuentemente apelan a distintos sentidos de "realismo" y, por consiguiente, fracasan a la hora de clarificar con precisión los problemas que están en debate" (p. 87).

El escrito está compuesto por tres secciones: en la primera se examinan los argumentos del profesor Wiltsche, especialmente su lectura de los parágrafos 24 y 52 del libro "Ideas I" de Husserl, que le permite a Wiltsche relacionarlo con el Empirismo Constructivo de Van Fraassen, es decir, que hace ver a Husserl como un antirrealista sofisticado. En la segunda sección se señalan las dificultades de la interpretación que hace Wiltsche de la obra de Husserl y se sugieren otros modos de comprender dichos textos. Finalmente, la tercera sección se dedica a considerar si el asunto de las entidades teóricas o inobservables tiene cabida en la fenomenología de Husserl, apostando por una respuesta afirmativa.

1 En varios artículos se aborda la relación de Husserl con el realismo o con el antirrealismo. A modo de ejemplo pueden considerarse los trabajos de Gutting (1978), Rouse (1987), Drummond (1988), Soffer (1990), Mormann (1991), Belousek (1998), Vallor (2009). La relación entre Husserl y la filosofía de la ciencia puede encontrarse en Aguirre (2011). De modo más general, Kochan (2011) y Kochan e Smith (2011) hacen una presentación de lo que se denominaría "Fenomenología de la ciencia". 


\section{Los argumentos de Wiltsche para adscribir a Husserl en el antirrealismo}

El eje del debate contemporáneo entre realismo y antirrealismo lo constituye la pregunta por el estatus de las entidades teóricas, también llamadas inobservables, esto es: iones, quarks, campos de fuerza y demás. Mientras que el realista se compromete con el estatus ontológico de estas entidades, el antirrealista considera que son simples ficciones que salvan la teoría o que no tenemos suficientes elementos para pronunciarnos acerca de su existencia o inexistencia. Esta última postura está más acorde con los postulados empiristas según los cuales sólo podemos obtener conocimiento de la experiencia sensible. Plantear una existencia que trascienda los datos sensibles, sería caer en posturas metafísicas. El argumento sería: puesto que no podemos tener experiencia directa de las entidades teóricas, no podemos afirmar que ellas existan; hacerlo sería exceder los límites de nuestras capacidades cognitivas y entrar en terrenos especulativos. Las entidades teóricas podrían ser, a lo sumo, ficciones útiles. El propósito de Wiltsche es alinear a Husserl entre aquellos filósofos que respaldan esta visión antirrealista acerca de las entidades teóricas o, por lo menos, mostrar que en Husserl hay una cierta tendencia antirrealista. Ahora bien, consciente de los varios tipos de antirrealismo, Wiltsche procura relacionar a Husserl solamente con el antirrealismo denominado Empirismo Constructivo, propuesto por el filósofo Bas van Fraassen. ${ }^{2}$

Es preciso caracterizar brevemente en qué consiste el Empirismo Constructivo. Este nombre fue usado por Van Fraassen (1980) para hacer frente a las tesis tanto del Realismo científico como de la doctrina positivista y, a su vez, para reivindicar el empirismo en la filosofía de la ciencia. Luego de hacer una revisión de las tesis de algunos realistas, Van Fraassen elabora una definición mínima del realismo: para el realista, "la ciencia se propone darnos, en sus teorías, un relato literalmente verdadero de cómo es el mundo; y la aceptación de una teoría científica conlleva la creencia de que ella es verdadera" (1980, p. 8. Énfasis en el original). Como alternativa al realismo, el

2 Es necesario advertir, de entrada, que Van Fraassen reconoce cierta deuda con Husserl en varias ocasiones; por ejemplo en la obra de Van Fraassen, de 2002, especialmente el apéndice B, 7. Igualmente, Van Fraassen está familiarizado con expresiones como "ir a las cosas mismas" (Van Fraassen, 1980), así como la distinción que hace Husserl en la "Idea de la Fenomenología" (1907/2011), entre "actitud natural" y "actitud filosófica" (Van Fraassen, 1994). Sin embargo, Van Fraassen rechaza la (supuesta) carga de metafísica que involucra Husserl en su "empirismo". No es extraño, por tanto, que el propio Van Fraassen vea el empirismo contemporáneo, léase el que él respalda, como superior a sus críticos, específicamente, a los idealistas británicos y a "Husserl, James, Dewey, Wittgenstein" (2007, p. 368). Una lectura detallada de Van Fraassen permitiría concluir que entre él y Husserl no podría haber encuentro sino más bien divergencia. 
Empirismo Constructivo tendrá que desafiar, por consiguiente, esta doctrina realista. Ante el primer enunciado, la idea de un relato literalmente verdadero, Van Fraassen sostendrá que "el lenguaje de la ciencia debería ser interpretado (construed) literalmente, pero sus teorías no necesitan ser verdaderas para ser buenas" (ibidem, p. 10); esta característica del Empirismo Constructivo le permite desligarse tanto del positivismo como del instrumentalismo. En consecuencia, si, por ejemplo, entre los enunciados de una teoría está: "hay electrones", la teoría dice que hay electrones. El punto en cuestión es el segundo enunciado, pues de asumir que el lenguaje de la ciencia debe ser comprendido literalmente no se sigue necesariamente que tengamos que creer en la verdad de la teoría: "todavía podemos decir que no hay necesidad de creer que las buenas teorías son verdaderas, ni creer ipso facto que las entidades que postulan son reales" (ibidem, pp. 11-12). Como corolario de su distancia con el Realismo Científico, Van Fraassen señala el presupuesto básico del empirista constructivo: "La ciencia se propone ofrecernos teorías que son empíricamente adecuadas; y la aceptación de una teoría involucra como creencia solamente que ella es empíricamente adecuada" (ibidem, p. 12. Énfasis en el original).

Una vez presentado el contexto en el que Wiltsche ubica a Husserl, paso a reconstruir de manera detallada sus argumentos, señalando de antemano que ellos se fundamentan, principalmente, en su interpretación de los parágrafos 24 y 52 de Ideas relativas a una fenomenología pura y una filosofía fenomenológica (en adelante "Ideas I"), de Husserl. Las líneas de argumentación empleadas por Wiltsche son: 1. lectura en clave empirista del "principio de todos los principios" (en adelante PP) de Husserl; 2. lectura del $§ 52$ de Ideas $I$ en consonancia con la lectura empirista de PP, con miras a considerar el asunto de las entidades teóricas; 3. relación de PP y el "principio de observabilidad" (en adelante PO) de Van Fraassen. Finalmente, Wiltsche concluye que Husserl puede leerse desde el Empirismo Constructivo de Van Fraassen, es decir, que Husserl es un antirrealista científico. A continuación, despliego estas líneas de argumentación.

En primera instancia, Wiltsche se dirige al $\$ 24$ de Ideas I, titulado "El principio de todos los principios" y de allí extrae la siguiente formulación de PP que, en las propias palabras de Husserl (1962), dice:

no hay teoría concebible capaz de hacernos errar en punto al principio de todos los principios: que toda intuición en que se da algo originariamente es un fundamento de derecho del conocimiento; que todo lo que se nos brinda originariamente (por decirlo así, en su realidad corpórea) en la "intuición", hay que tomarlo simplemente como se da, pero también solo dentro de los límites en que se da (p. 58, énfasis en el original). 
PP es reformulado por Wiltsche (2012) de la siguiente manera:

[PP:] Puede decirse que $\mathrm{X}$ se da intuitivamente si una intención hacia $\mathrm{X}$ se cumple por la presencia directa, inmediata de X. Así pues, la intuición es una experiencia intencional en la que la referencia a $\mathrm{X}$ no está mediada por presupuestos que no son parte de la cosa misma intencionada (p. 108).

Como la intención de Wiltsche es relacionar PP con el empirismo, extrae la siguiente consecuencia: "la intuición, es decir, la congruencia entre la intención-de-la-cosa y la donación real (actual) de la respectiva cosa, establece la norma ideal para todas las empresas justificadoras" (2012, p. 109). De acuerdo con esto, para Husserl, una justificación ocurre sólo cuando se da la justificación inmediata; en otras palabras, solo cuando la cosa sea intuitivamente dada tal y como fue intencionada, puede completarse legítimamente la cadena indefinida de justificaciones mediatas. Llegados a tal justificación inmediata, "está justificada, más allá de toda duda razonable, mi creencia acerca de su situación" (idem). En consecuencia, sólo a partir de tal intuición puedo afirmar: "conozco". Valga agregar que, según el Husserl de Wiltsche, esta intuición no requiere justificación.

Puede verse cómo la primera estrategia de Wiltsche consiste en identificar el concepto husserliano de intuición con la presencia directa, inmediata de X. De este modo, PP estaría en consonancia con el dictum empirista según el cual el fundamento de derecho del conocimiento se desprende de las impresiones y solamente en los límites de estas impresiones.

En segunda instancia, Wiltsche aborda el §52 de "Ideas I": "Complementos. La cosa física y la "causa desconocida de las apariencias", ${ }^{3}$ a partir del convencimiento de que este parágrafo es la respuesta de Husserl a la cuestión de "si los términos teóricos pueden usarse o no para referirse a las capas inobservables del mundo físico" (Wiltsche, 2012, p. 110). El fragmento en el que va a respaldar su conjetura es el siguiente:

Habría que señalar, también, cómo una explicación de los procesos dados en la percepción por medio de realidades, en sentido estricto, causales e hipotéticamente admitidas, por medio de cosas desconocidas (como, por ejemplo, la explicación de

3 En la versión inglesa de la obra, usada por Wiltsche en su artículo, aparece un poco más explícito el título del parágrafo. Allí se lee: "Supplementations. The Physical Thing as Determined by Physics and the "Unknow Cause of Appearances" (Husserl, 1983, p. 118). Creo que la versión española de Gaos es más fiel al original alemán donde se lee: Ergänzungen. Das physikalische Ding und die "unbekannte Ursache der Erscheinungen" (Husserl, 1913, p. 97), aunque la versión inglesa recoge mejor lo fundamental de lo tratado en el parágrafo. Las referencias a esta obra considerarán las tres versiones, citando preferentemente la versión castellana. 
ciertas perturbaciones planetarias por la hipótesis de la existencia de un nuevo planeta todavía desconocido, Neptuno) es en principio algo distinto de una explicación en el sentido de la determinación física de las cosas de experiencia sirviéndose de medios de explicación física de la índole de los átomos, los iones, etc. (p. 120, énfasis mío)

Una vez adoptada la lectura empirista de PP, este párrafo no sería sino la confirmación de tal lectura. En primer lugar, pareciera que Husserl mismo está estableciendo una distinción de principio entre los objetos (actual o potencialmente) observables a ojo desnudo (planetas) y los inobservables (átomos o iones), toda vez que sugiere una diferencia entre una "percepción por medio de realidades, en sentido estricto, causales e hipotéticamente admitidas", y aquellas que pertenecen a "una explicación en el sentido de la determinación ${ }^{4}$ física de las cosas de experiencia". En segundo lugar, al aplicar PP tal y como lo lee Wiltsche, esta diferencia en principio radicaría en que tanto la existencia ${ }^{5}$ como el conocimiento de los primeros estarían garantizados (por ser una percepción en "sentido estricto", esto es, acorde con PP), mas no así las de los segundos (que estarían circunscritos a la teoría que explique el modo como la ciencia, por ejemplo la física, determina físicamente las cosas de experiencia). Como conclusión, según Wiltsche, Husserl podría respaldar la literalidad de los términos teóricos a la hora de referirse a las capas inobservables del mundo físico, mas no se comprometería con las realidades de los mismos, ya que no hay modo de cumplir PP; a lo sumo dirá que son útiles al momento de explicar lo físico.

Es en este punto en el que Wiltsche relaciona PP de Husserl con PO de Van Fraassen (1980), el cual se enuncia así: “[PO:] X es observable si hay circunstancias tales que si $\mathrm{X}$ está presente a nosotros bajo esas circunstancias, entonces lo observaríamos" (p. 16).

En el caso enunciado por Husserl, Neptuno podría cumplir PO, mas átomo o ion no correrían con igual suerte. ¿A qué se debe esto? Siguiendo al pie de la letra las explicaciones de los antirrealistas, Wiltsche está convencido de que, aunque parezca improbable, no es imposible afirmar que podríamos observar directamente a Neptuno; es decir, "no hay razones para negar que Neptuno es potencialmente observable" (2012, p. 111); sin embargo, a Wiltsche, como a

4 El sentido del concepto husserliano de "determinación" se aborda en el siguiente apartado.

5 Wiltsche no compartiría esta afirmación, pues, como lo advierte, su versión "sofisticada" de antirrealismo no es ontológica sino epistemológica. Al igual que Van Fraassen (2001), la actitud recomendada acerca de la realidad de los inobservables es el agnosticismo. Sin embargo, en tanto se intentará confrontar esta interpretación antirrealista, se asume que lo que está en juego implica tanto problemas ontológicos (la realidad de los inobservables), como epistemológicos (el conocimiento verdadero de los aspectos inobservables del mundo transmitido por las teorías científicas). 
la mayoría de los antirrealistas, le parece que no es aplicable esto mismo a los átomos o a los iones, toda vez que estas entidades parecen exceder nuestro alcance epistémico. Para aclarar su explicación, Wiltsche establece la siguiente distinción con respecto a las entidades: en primer lugar están las "entidades observables" que, en la actual filosofía de la ciencia se denominan: objetos de tamaño medio; en segundo lugar, están las "entidades que son inobservables ahora pero no inobservables en principio" (por ejemplo, galaxias distantes); finalmente, "entidades que son en principio inobservables" (Cf. 2012, p. 111). Obviamente, Wiltsche dirá que la fenomenología se compromete sólo con el tipo de entidades 1 y 2; más aún, afirmar en fenomenología la existencia de las entidades del tipo 3 sería un contrasentido.

La conclusión que extrae Wiltsche después de estas consideraciones es que la fenomenología de Husserl está en simetría con el Empirismo Constructivo de Van Fraassen, especialmente en los siguientes ítems: 1. las teorías científicas, cuando hacen referencia a los inobservables, se adoptan en el sentido de adecuación empírica, mas no de verdad por correspondencia; 2. la noción de verdad solo es aplicable a las entidades observables; 3. el antirrealista no afirma la inexistencia de los inobservables; simplemente suspende el juicio pues no hay suficientes razones para creer en la realidad de los inobservables.

\section{Dificultades de la lectura de Husserl en clave antirrealista}

En este apartado se intenta confrontar los argumentos dados por Wiltsche para fundamentar el supuesto antirrealismo de Husserl. El objetivo inicial será desligar de PP, al menos en la consideración de la cosa física, la carga de empirismo que Wiltsche infiere de $\operatorname{los} \S \S 24$ y 52 de "Ideas I". En el apartado final se propondrá que no es un contrasentido hablar de entidades teóricas o inobservables en la fenomenología husserliana. Para lograr el primer objetivo, habrá que recurrir a cierta terminología de Husserl, la cual, debido a su complejidad, será apenas indicada en sus aspectos más básicos, toda vez que una exposición amplia de la misma exigiría desarrollos que exceden los propósitos del presente artículo.

El primer argumento de Wiltsche se fundamenta en que estaríamos plenamente justificados para hablar del conocimiento de un objeto si un juicio sobre ese objeto ("una intención hacia X") se corresponde ("se cumple") con ese objeto; sin embargo, habría una condición más: que ese objeto ("cosa misma") se presente de manera directa o inmediata. Tal y como también se ve en su interpretación del $\S 52$, esa presencia debe ser sensorial, es decir, 
debe cumplir PO; de lo contrario, el problema de los inobservables se haría irrelevante. La cuestión, entonces, consiste en saber si el "darse originario" (u originariamente) tiene que restringirse al darse real o posible en la percepción sensible.

Lo primero que se advierte en este argumento es que no está en consonancia con el contexto en el que Husserl enuncia PP (\$24), contexto que comprende $\operatorname{los} \S \S 18$ a 26 de "Ideas I", agrupados bajo el título: "Erróneas interpretaciones naturalistas". Precisamente, la reacción de Husserl es contra el empirismo (§18) y, más específicamente, contra su pretensión de "identificar o confundir la fundamental exigencia de un volver a las "cosas mismas" con la exigencia de fundar todo conocimiento en la experiencia. Dada la comprensible limitación naturalista del marco de las cosas "cognoscibles", para el empirista pasa sin más la experiencia por el único acto en que se dan las cosas mismas" (Husserl, 1962, p. 49). Esta identificación, que implica que las cosas que se presentan y se dan a la comprensión sin problema, deja de lado, sin verlas, distinciones que deben darse con la más clara evidencia intelectual (§19).

De esto puede inferirse que no es posible identificar el llamado fenomenológico a las cosas mismas con la apelación empirista a la experiencia como tribunal definitivo de toda pretensión de conocimiento; ${ }^{6}$ además, que en el marco del empirismo se suprimen distinciones que son fundamentales para el fenomenólogo, por ejemplo: "las regiones fundamentales de objetos $\mathrm{y}$, correlativamente, los tipos regionales de intuiciones en que se da algo; los correspondientes tipos de juicios y, finalmente, las normas noéticas que requieren como fundamento de los juicios de tales tipos justo esta clase de intuición y no otra...” (Husserl, 1962, p. 49). No habría, entonces, una intuición limitada a la experiencia sensorial; en tal sentido, el darse originario no se reduce al ver empírico: "La "visión" directa, no meramente la visión sensible, empírica, sino la visión en general, como forma de conciencia en que se da algo originariamente, cualquiera que sea esta forma, es el último fundamento de derecho de todas las afirmaciones racionales" (Ibidem, p. 50. Énfasis en el original). Finalmente, en cuanto a las intuiciones de la experiencia sensible, a diferencia de lo que supone Wiltsche, estas no están per se garantizadas, más aún, el ver sensible es "el ver por esencia "imperfecto" (idem), teniendo que agregarse o disminuirse "fuerza". No es extraño, por consiguiente, que "una afirmación que posea en la experiencia un fundamento de derecho directo y por tanto auténtico, sin embargo tenga en el curso de la experiencia que ser verdad exclusivamente a los observables" (p. 113). 
abandonada en virtud de un derecho opuesto de esta que prepondere sobre él y lo anule" (idem). ${ }^{7}$ En consecuencia, PP debe comprenderse en un sentido más allá del marco meramente empirista de la "presencia directa, inmediata de X". Puede verse que la interpretación de Wiltsche fracasa al confundir "la intuición en que se da algo originariamente" con "la presencia directa, inmediata de X". Entonces, el asunto será desentrañar, de manera sintética, en qué consiste la intuición para Husserl. Como varios conceptos husserlianos, este fue tomado de la tradición (en especial de la escolástica); por tanto, puede conducir a interpretaciones erróneas si se lee sólo en el contexto de tal tradición. En efecto, tal y como lo sostiene Hintikka (2003), luego del debate entre innatistas y empiristas, el concepto de intuición "se convirtió en poco más que un sinónimo de inmediatez" (p. 171). Fue con Kant con quien la intuición alcanzó una interpretación especial: para él, "las intuiciones en el sentido mínimo de la palabra no son sino representaciones singulares que se distinguen de los conceptos generales. La intuición no era una fuente de verdades o aprehensiones, sino sólo el medio para representar particulares" (Hintikka, 2003, p. 171). Podría decirse que el sentido primario de intuición que adopta Husserl es tanto pre-kantiano como kantiano, es decir, que intuir es tener un conocimiento inmediato de cualquier tipo y, además, que la intuición husserliana, como la kantiana, no da estatus especial al conocimiento intuitivo, más allá de la atención que debe brindarse a lo inmediatamente dado a nosotros en la experiencia.

Si bien Wiltsche recurre a este sentido primario de intuición, ha olvidado que en el propio Husserl, como ya se mencionó, hay diferencia entre la "visión directa" y la "visión en general" como "forma de conciencia en que se da

7 Quizás por la atención que se presta a la intuición de esencias, en ocasiones se deja de lado lo que Husserl denomina en "Ideas I": "percepción de cosas" (Dingwahrnehmung) (§44). Es muy importante mencionar que Husserl ha desarrollado una Teoría de la Abschattung (escorzo, matiz, perspectiva), la cual permite poner en cuestión la pretendida legitimación del conocimiento que brota de la percepción sensible, pues esta se caracteriza por una esencial inadecuación. Para Husserl (1962), "Una cosa se da necesariamente en meros "modos de aparecer", en que necesariamente hay un núcleo de algo "realmente exhibido", rodeado, por obra de apercepciones, de un horizonte de algo "co-dado" impropiamente y más o menos vagamente indeterminado" (p. 99). Ahora bien, no se trata de postular una inadecuación o indeterminación que conduzca al escepticismo (a lo que podría conducirnos la propuesta antirrealista); al contrario, Husserl defiende que "la indeterminación significa [...] necesariamente la posibilidad de determinarse en un estilo rigurosamente prescrito" (idem). Esta mención a la Teoría de la Abschattung permite sostener que una percepción de objetos que pretenda establecer la adecuación en los estrechos márgenes de la percepción sensible, necesariamente está destinada al fracaso, toda vez que la percepción de objetos es, por esencia, inadecuada. Por tanto, aunque las entidades teóricas sean del tipo de entidades vagamente indeterminadas, tanto el científico natural como el fenomenólogo tienen el deber de determinar rigurosamente (lo que no significa: de modo infalible), como en efecto lo hacen, la cosa percibida, mas no ya en el terreno exclusivo de la percepción sensible. Una alternativa se planteará cuando se mencione la intuición de esencias. 
algo originariamente" (§19); así pues, es posible postular ese sentido primario de intuición, mas no es preciso limitarlo a él. Habría que señalar el modo como Husserl plantea el acceso a esa forma de conciencia en que se da algo originariamente. La fenomenología es el empeño por esclarecer este asunto a través de la detallada exposición de las reducciones fenomenológicas. El objetivo será mostrar a continuación que las intuiciones no se limitan a lo que se da en la percepción sensible (como lo requiere el empirismo), sino que hay intuiciones que se descubren a través de reducciones fenomenológicas, señalando otro sentido de intuición no tenido en cuenta por Wiltsche.

Aunque el texto más ampliamente considerado por Wiltsche es Ideas $I$, bien es sabido que Husserl postuló muy pronto una distinción entre el pensamiento natural y el filosófico; entre la actitud natural y la ciencia, y la actitud intelectual filosófica (reflexiva). Ya en 1907, señalaba que la característica de la primera actitud es que "no se muestra todavía preocupada por la crítica del conocimiento. Cuando nos hallamos en esa actitud, nuestra atención está dirigida intuitiva ${ }^{8}$ y meditativamente a las cosas que en cada caso no están dadas, y dadas de manera evidente [...]" (Husserl, 2011, p. 75). Al afirmar que todavía no hay una crítica en este tipo de actitud, se nota que es necesaria otra actitud que asuma tal crítica, esto es, que supere el carácter enigmático del conocimiento. Ciertamente, Husserl propone un cambio de actitud que se encargue de "resolver los problemas concernientes a la correlación de conocimiento, sentido del conocimiento y objeto del conocimiento investigando la esencia del conocimiento" (ibidem, p. 81). Es por ello que se da a la tarea de formular el método que aclare tanto la esencia del conocimiento como la objetualidad del mismo. En esto consiste primeramente la tarea del fenomenólogo. ${ }^{9}$

Tanto el cambio de actitud (de la natural a la filosófica) como el acceso a nuevas regiones del ser se dan a partir de un conjunto de "reducciones progresivas" que parten de un ejercicio voluntario de un sujeto, de un intento por colocar entre paréntesis o poner fuera de juego (técnicamente llamado por

8 El término usado por Husserl en esta frase es: anschauend. De acuerdo con el "Glosario-Guía para traducir a Husserl", este término, en una de sus acepciones dice: "que ve". Por el contexto en el que Husserl utiliza esta palabra, se ratifica que hay un tipo de intuición empírica. También en "Ideas l", Husserl, haciendo referencia a esta actitud natural, usa los términos anschaulich y anschauungsfeld para referirse respectivamente a que en esta actitud el mundo se da "intuitivamente" y que tanto los objetos como los seres animados se dan en mi "campo de intuición". Podríamos conceder a Wiltsche que, en efecto, Husserl usa la palabra intuición en el sentido que él quiere adscribirle. El reto ahora es mostrar que la intuición no se limita a esta "presencia directa, inmediata de X".

9 Aunque no puede afirmarse que "la fenomenología de Husserl se limite o pueda reducirse a este problema [del conocimiento]", sino que "la fenomenología del conocimiento se sugiere de modo preciso para esclarecer las características del procedimiento fenomenológico [...]" (Ströker, 1997, pp. 28-29). 
Husserl epojé) la tesis general de la actitud natural, esto es, el conceder que "la "realidad" la encuentro [...] como estando ahí delante y la tomo tal como se me da, también como estando ahi" (Husserl, 1962, p. 69). El resultado de tal puesta entre paréntesis, los "residuos fenomenológicos", abre un nuevo campo de exploración que posibilita considerar a la conciencia como tema, es decir, explorar las "vivencias puras", la "conciencia pura" con sus "correlatos puros", el "yo puro" (ibidem, p. 75). Hay que resaltar, sin embargo, que Husserl es insistente en advertir que en ningún momento hay pérdida del mundo o aniquilamiento de la realidad, solo que tal realidad se inserta en la conciencia, siendo una vivencia de conciencia (cogitatio o noesis), conservándose la realidad misma, "con sus propiedades objetivas, su extensión en el espacio, su situación objetiva a esa cosa espacial que se llama mi cuerpo" (Husserl, 1962, p. 79); mas no ya en actitud natural sino fenomenológica, a modo de cogitatum o noema. En este punto cabe destacar el rol tan preponderante que juega en Husserl su formulación de la intencionalidad, ${ }^{10}$ a partir de la cual es posible comprender que "el mundo pertenece esencialmente a la relación intencional; mundo y conciencia se pertenecen; objeto y sujeto están inseparablemente unidos en una unión original y originante, pues solo a partir de esa unión se puede hablar de objeto y de sujeto" (San Martín, 1986, p. 234).

El punto de separación con Wiltsche, así como con los empiristas de cualquier tipo, parte de lo siguiente: "la intuición [...] puede pertenecer a los universales, tales como formas o esencias, y no solo a los particulares" (Hintikka, 2003, p. 176). En el terreno de la conciencia pura, alcanzado mediante las reducciones, es posible encontrar, por ejemplo, otro tipo de intuición llamado categorial: "que se refiere a "una clase de donación", un caso de ver real (actual), pero que se da ahora como un asunto conceptual más que empírico. Puesto que esas categorías no son objetos de ver literal, el uso husserliano de "ver" es meramente metafórico" (ibidem, p. 177). Lo relevante es que no habría una escisión entre ambas clases de intuición, ya que la intuición, entendida en el sentido primario, provee los cumplimientos de nuestros noemata, lo que implica que yo no elijo los cumplimientos, sino que ellos se me dan en la experiencia; de igual forma, los cumplimientos son una cuestión objetiva: literalmente la realidad incide en mi conciencia (ibidem, p. 179). Sin embargo, pese al rol fundamental del primer sentido de intuición, el análisis fenomenológico le permite al investigador dirigir su atención no a los datos hyléticos, sino que se puede dirigir, en el segundo sentido de intuición, 
a las formas o esencias impuestas sobre ellos: "esto presupone que un analista puede separar en la conciencia las formas (eide) de lo material en el que ellas se incrustan, convertirlas en objetos de otra clase, y dirigir su atención hacia ellos. Esto es lo que la Wesensschau de Husserl se propone alcanzar. Esta intuición tiene el efecto de abrir a mi conciencia, no solo un objeto empírico que consiste de materia y forma, sino esa forma o esencia en si misma, separada de su hyle" (ibidem, p. 180).

El breve recorrido por el concepto husserliano de intuición, y por lo que este implica en el contexto de las reducciones, permite afirmar algunas consecuencias que harían difícil la lectura antirrealista del 24 , propuesta por Wiltsche: en primer lugar, Husserl no limita el concepto de intuición a la percepción sensible, al ver empírico ("presencia inmediata de X"), el cual acaece en la actitud natural. En segundo lugar, Husserl sugiere suspender tal ver empírico en aras de alcanzar una intuición esencial o visión de esencias (Wesensschau). En tercer lugar, la visión de esencias, no dependiente de la hyle, permite considerar la realidad, no como meros fantasmas o ficciones de esta. En cuarto lugar, si bien las percepciones sensibles juegan el rol de evaluar el cumplimiento o no de las vivencias de conciencia (noemata), lo que no es dado a la intuición en el sentido primario (percepción sensible) puede ser descubierto o constituido a partir de reducciones fenomenológicas. Esto último no significa, sin embargo, que sea construido en la conciencia, sino como una manera particular en que un sujeto descubre el sentido de un objeto. ${ }^{11}$ En conclusión, no puede leerse PP en el estrecho margen del empirismo, pues la intuición, el darse originario, incluye el sentido primario (al que parece limitarse Wiltsche), pero también, y sobre todo, la intuición esencial.

Si bien la primera estrategia de Wiltsche ha quedado debilitada, creo que también la segunda estrategia lo estará, en tanto se respalda totalmente en PP. A continuación se retará la interpretación que Wiltsche hace del $§ 52$ de "Ideas I", según la cual es posible afirmar que en Husserl hay una distinción de principio entre las entidades teóricas y las entidades observables, lo que alinearía a Husserl con los postulados de los antirrealistas científicos contemporáneos, en especial con Van Fraassen.

Lo primero que habrá que indicar es que el $\$ 52$ no está dedicado stricto sensu a la distinción entre entidades teóricas y observacionales, pese a lo que

11 Aquí hay un distanciamiento profundo entre Van Fraassen y Husserl. Mientras el primero habla de Empirismo Constructivo, el segundo habla de Fenomenología constitutiva. Es en esta fenomenología, donde podría rescatarse un sentido nuevo de realismo (entendido en el contexto de la aún confusa concepción del Idealismo Trascendental de Husserl), toda vez que permite establecer "las condiciones de posibilidad del conocimiento de la realidad objetiva" (Bernet, 2004, p. 2). 
presume Wiltsche. Este parágrafo comienza estableciendo las características de la cosa física (entendida en el sentido de "la cosa determinada por la física", o por "los investigadores de la naturaleza"), lo cual se hace en contraposición a lo que Husserl denomina: "la cosa de la imaginatio sensible". De esta comparación surgen tres formulaciones en negativo: 1. Si bien la cosa física se presenta al científico como "mera apariencia" o algo "meramente subjetivo", no puede confundirse con la "subjetividad de las vivencias", con meras "vivencias". 2. La cosa que aparece tampoco es una "falsa apariencia" o una "imagen" defectuosa de una "verdadera" cosa física. Finalmente, 3. tampoco se pueden tomar las determinaciones que aparecen como "signos" de las verdaderas determinaciones. ${ }^{12}$ La crítica de Husserl va dirigida hacia el planteamiento (¿kantiano?) según el cual habría una realidad incognoscible (que sería la verdadera y) que respalda las apariencias o fenómenos a los cuales tenemos acceso; en tal sentido, jamás podríamos acceder a esa realidad ni a su verdad; a lo sumo conoceríamos tales apariencias e ignoraríamos las causas que las respaldan.

Sin embargo, a mi modo de ver, el trasfondo auténtico del $\S 52$ lo constituye la relación que se da entre la cosa (determinada por la) física y el "ser que se da en persona en la "percepción" misma" (Husserl, 1962, p. 121). Es claro que para Husserl no son lo mismo: por un lado, la cosa física, "la "cosa de que tenemos propiamente experiencia" nos da el "mero esto ", una "x vacía", que se torna el soporte de las determinaciones físicas exactas, que no caen ellas mismas dentro de la experiencia propiamente tal “ (idem). Así pues, la ciencia

12 Como puede verse, el concepto Bestimmung, traducido al español como "determinación", está muy presente en este parágrafo. Es necesario decir algo respecto a eso, toda vez que podría dar lugar a malas interpretaciones que favorecerían una perspectiva antirrealista sobre las teorías científicas. A modo de ejemplo, puede enunciarse la perspectiva de Cartwright (1983), para quien no hay relación entre teoría y realidad, sino que tal relación se despliega a través de la siguiente ruta: de la teoría al modelo y del modelo a la ley fenomenológica; en consecuencia, si bien las leyes fenomenológicas describen con relativo éxito la realidad, esta es determinada al modo de adecuación descriptiva, pues "las poderosas leyes explicativas que se encuentran en la física teórica no establecen la verdad" (p. 3). Si bien Wiltsche interpretaría el Bestimmung husserliano en el marco de la física como el pronunciamiento sobre la realidad sin pretensiones de afirmar o negar lo no dado fenoménicamente (en el contexto, digamos, de Ernst Mach), Bestimmung en Husserl no adopta dicho sentido, toda vez que, aplicadas las reducciones, puede distinguirse entre "determinaciones" (o meros signos) (Erscheinungsbestimmtheit) y "verdaderas determinaciones" (wahren Bestimmtheiten). Estas últimas serían las que permiten, tal y como una breve revisión de Ideas I sugiere, "la última determinación del sentido (Sinnesbestimmung) del "ser" de sus objetos" (§62); esto es, las que permiten que lo dado en la indeterminación sea "susceptible de determinación (Bestimmbarkeit)" (\$69); lo que, en últimas, no es sino la posibilidad no de un conocimiento empíricamente adecuado, sino de un conocimiento en el que se tengan presentes las "formas esenciales del dominio y sus determinaciones esenciales (Wessensbestimmungen), deduciéndolas mediante una aplicación consecuente de los axiomas” (§73). En cualquiera de los casos enunciados, Husserl está abogando por algo más que Erscheinungsbestimmtheit (determinación de lo que aparece), apartándose de la interpretación de la teoría como mera adecuación empírica (tal como lo defiende Cartwright y Van Fraassen). 
de la naturaleza no se detiene en los cambiantes modos de aparición de las cosas, sino "lo que de idéntico aparece en la cambiante continuidad de estos modos de aparecer" (ibidem, p. 122). Sin embargo, tanto las cosas sensibles como la cosa que el físico observa están en íntima relación:

la cosa que el físico observa, con la que experimenta, que está viendo constantemente, toma en la mano, pone en la balanza, mete en el horno, esta cosa y no otra es la que se convierte en sujeto de los predicados físicos, como son el peso, la masa, la temperatura, la resistencia eléctrica, etc. Igualmente son los procesos y relaciones percibidos mismos los determinados por medio de conceptos como fuerza, aceleración, energía, átomo, ion, etc. (idem, énfasis mío)

De acuerdo con esto, Husserl parece estar postulando, para la ciencia de la naturaleza, no una relación uno-a-uno entre la cosa sensible y la cosa (determinada por la) física, sino que está señalando el estrecho vínculo entre una y otra, vínculo que, en consonancia con el tratamiento que se dio al concepto de intuición, no puede quedar reducido a la percepción sensible. Más aún, Husserl es claro en plantear que el físico "está obligado a practicar ciertos modos de apercepción, a llevar a cabo ciertas construcciones intencionales como requeridas por la razón, y a llevarlas a cabo para determinar teóricamente las cosas de la experiencia sensible" (ibidem, p. 123). En consecuencia, la teorización del científico natural debe estar soportada por la realidad; sin embargo, esto no implica que la realidad se restrinja a las cosas sensibles (imaginatio sensible), más bien a la intellectio física, terreno en el cual "brotan todos los productos mentales, ontológicos e ideales que se expresan en los conceptos físicos y sacan, y deben sacar, su sentido, exclusivamente del método de la ciencia natural" (idem).

¿Por qué Wiltsche sitúa la discusión de este parágrafo en el debate sobre los inobservables? Según puedo inferir, Wiltsche está persuadido de que el PP de Husserl coincide con el PO de Van Fraassen. Esta seguridad lo lleva a leer ciertas expresiones confusas de Husserl (v. gr. "la cosa percibida misma", darse "en persona", "realidad corpórea") en el contexto del empirismo; igualmente, a adoptar discusiones que Husserl señala a modo de ejemplo -tal es el caso de la distinción en principio entre Neptuno y los átomos o iones-, no tanto para comprometerse con ellas, como sí lo hace Van Fraassen, sino para indicar cómo ellas están necesitadas de clarificación; en tal sentido, las deja a un lado para "reanud[ar] la marcha", transitando por caminos más seguros para el fenomenólogo. 
En el punto específico de los inobservables, por ejemplo, Husserl no recomendaría el agnosticismo o escepticismo ${ }^{13}$ sugerido por Van Fraassen (2001) y Wiltsche, toda vez que esta es la característica típica de la ciencia dogmática (§26), mientras que Husserl reclama una "actitud específicamente filosófica". Además, si bien Husserl estaría en desacuerdo al tomar los inobservables como ficciones útiles o construcciones sociales, ${ }^{14}$ tampoco exigiría una presencia como la que exige la imaginatio sensible; en tanto entidades postuladas por la ciencia natural, los inobservables entrarían a la esfera de la intellectio física. Es preciso dejar claro que todo el $§ 52$ es un intento por mostrar cómo imaginatio sensible e intellectio física están en íntima relación, en la medida en que "la cosa percibida misma, [es] siempre y por principio, exactamente la cosa que el físico investiga y determina cientificamente" (Husserl, 1962, p. 121. Énfasis en el original). El siguiente apartado, espero, permitirá superar esta aparente paradoja.

\section{Una aproximación a los inobservables desde la fenomenología de Husserl}

Este apartado final se propone mostrar que la posibilidad de los inobservables en ciencia no es ajena al modo como Husserl ve el quehacer científico. En primer lugar, presentaré algunas características de la ciencia, tal y como la define Husserl; posteriormente, señalaré cómo para Husserl la postulación de entidades inobservables, respaldada por instrumentos, es propia de la praxis científica, y contribuye al logro de la evidencia exigida a los "juicios justos" o enunciados científicos verdaderos.

De modo general, Husserl (1995) define el saber como la posesión de la verdad, siendo entendida esta como "objeto de un juicio justo" (p. 41) más evidencia, esto es: "luminosa certeza de que lo que hemos reconocido es, o lo que hemos rechazado no es" (idem). A diferencia de lo que muchos piensan, certeza no es ni convicción ciega, ni opinión vaga o infundada, sino que debe estar respaldada por "ciertas "notas", que nos notifican la existencia de la

13 Sankey (2008), al presentar la versión del empirismo de Van Fraassen, la denomina "escéptica con relación a la teoría del conocimiento" y continúa: "De acuerdo con tal antirrealismo escéptico, las afirmaciones teóricas acerca de las entidades inobservables pueden corresponder bien con la realidad, pero la evidencia empírica nunca puede ser suficiente para apoyar la verdad de tales afirmaciones que pueden ser aceptadas, a lo mejor, como empíricamente adecuadas o garantizadas" (p. 12). En tal sentido, el supuesto agnosticismo no es más que un escepticismo, tendencia fuertemente rechazada por Husserl desde sus inicios (véase, por ejemplo, Husserl (1995), especialmente el Tomo I: "Prolegómenos").

14 Como lo toman algunas ramas de los recientes Estudios Sociales de la Ciencia, véase el caso de Latour e Woolgar (1986). 
situación objetiva admitida, o la justeza del juicio pronunciado" (ibidem, p. 42). Es notorio que Husserl no está exigiendo, para que haya conocimiento científico, una evidencia que implique "una conciencia inmediata de la verdad misma" (idem), lo cual ocurriría en pocos casos o en casos ideales; en cambio, en consonancia con su Teoría de la Abschattung, habla de ciertas notas que respaldarían con mayor o menor probabilidad tal certeza. Husserl denomina a este tipo de evidencia como: "la evidencia de la probabilidad" (EP); ahora bien, EP de una situación objetiva $A$ no funda la evidencia de su verdad; pero funda esas valoraciones comparativas y evidentes, por virtud de las cuales logramos distinguir, según los valores positivos o negativos de probabilidad. En consecuencia, Husserl no considera que la ciencia pueda siquiera alcanzar evidencia absoluta, tan solo puede obtener evidencia probable de un grado mayor o menor.

No significa con esto que Husserl está confundiendo verdad (Wahrheit) con probabilidad. Es claro que desde sus inicios, en su discusión con el psicologismo (y por extensión con el relativismo) defendió que la verdad es en sí, absoluta y tiene valor a priori; posteriormente, refina su concepción hasta plantear que la verdad es una "idealidad supra empírica" (überempirische Idealität) (Palette, 2010). Si bien podría entenderse el concepto de probabilidad en el contexto del tradicional concepto de verdad como adecuación y, por tanto, en el contexto de la verificación, esto es: comparar alguna representación (en la mente) con algún estado de hechos fuera de la mente (no aprehensibles por fuera de la representación), es necesario advertir que Husserl somete tal verdad por adecuación a su concepción fenomenológica, a saber: "la verdad es una experiencia, un reconocimiento específico (aunque no es necesario que se tematice de modo específico) de la coincidencia entre lo que es significado y lo que es dado" (Moran, Cohen, 2010, p. 335). El concepto clave aquí es el de coincidencia (Deckung). Con este concepto, Husserl está haciendo referencia a la relación entre una intención y su cumplimiento: cuando un acto de intención se cumple hay una coincidencia entre el acto intencionado y el acto cumplido. Moran e Cohen (2010) plantean el siguiente ejemplo para ilustrar el concepto: "si pierdo las llaves de mi carro y estoy buscándolas, entonces el acto intencional es el buscar las llaves de $m i$ carro. El acto de cumplimiento consiste en encontrar, precisamente, tales llaves. La coincidencia viene cuando reconozco las llaves que encuentro como las que estaba buscando (y no, por ejemplo, las llaves de otro carro que no me pertenecen)" (p. 66). La importancia del concepto radica en que una coincidencia completa sería un 
ideal, "con frecuencia, las intenciones solo se pueden cumplir parcialmente" (idem). ${ }^{15}$

Una característica relevante del modo como Husserl describe la ciencia es la recurrencia de esta a "artificios metódicos" (1995, p. 43); prácticamente esta es una característica esencial de la ciencia. ${ }^{16}$ Para Husserl, en la ciencia "hay innumerables proposiciones verdaderas, de cuya verdad solo nos percatamos cuando las hemos "fundamentado metódicamente"; es decir, que en estos casos y con respecto meramente a la proposición pensada, hay, sí, juicio, pero no hay evidencia" (idem). El hecho de que no tengan evidencia, no impide que sean consideradas en el cuerpo del teorizar científico; más aún, solo mediante este tipo de proposiciones puede superarse el ámbito de lo trivial o inmediatamente evidente, pudiendo acceder al conocimiento de verdades o probabilidades "que de otro modo permanecerían ocultas" (idem). Se tiene, entonces, que los artificios metódicos son parte esencial de la ciencia y del progreso del conocimiento científico. Podría pensarse que, en tanto los "Prolegomena" están dedicados fundamentalmente a la matemática, Husserl estaría hablando solo de métodos algorítmicos; sin embargo, aunque de pasada, Husserl también menciona un tipo de métodos denominados "métodos literalmente mecánicos", entre los cuales resalto: "los múltiples métodos para determinar la posición de una estrella, una resistencia eléctrica, una masa inerte, un exponente fraccionario, las constantes de la gravedad terrestre, etc. " (1995, p. 49); a partir de la aplicación de estos métodos y sus correspondientes "dispositivos", el científico prueba la validez objetiva de un juicio particular. En consecuencia, la ciencia opera con juicios probables, es decir, que pueden ir ganando con el paso del tiempo mayor grado de evidencia; asimismo, la

15 En el "Husserl-Lexikon" se hace una presentación más técnica del concepto, resaltando que la coincidencia se da mediante distintas síntesis: I. La síntesis de coincidencia (Deckung) como continuidad entre la cosa y la peculiaridad que la cosa tiene; II. La síntesis de coincidencia entre una percepción y un recuerdo de la misma con carácter discreto; y, III. Una síntesis de coincidencia explicativa que toma tanto los momentos continuos como los discretos (Serra, 2010, pp. 55-57). La consideración mínima de este concepto permite concluir, al menos, lo siguiente: Husserl da un papel relevante al acto intencional, pero este acto debe coincidir, mediante distintas síntesis, con un acto de cumplimiento. Además, es posible hablar en Husserl de un conocimiento con evidencia probable, probabilidad que aumentaría en los casos en los que los actos intencionales coincidan cada vez más con actos de cumplimiento; por ende, la evidencia de probabilidad podría ir alcanzando mayor precisión o exigir su abandono. En el contexto de discusión que nos ocupa, el concepto Deckung es esclarecedor, pues si bien las entidades teóricas son inobservables en el momento, no podría postularse esta inobservabilidad en principio, pues, mediante la creatividad e ingenio del científico, es posible alcanzar cumplimientos antes insospechados. En este caso, se puede dar la coincidencia entre un acto intencional -por ejemplo: la postulación de los quarks-, que encontraría sus respectivos cumplimientos -por ejemplo, la conclusión a la que llega Hacking (1983): "si se pueden rociar, entonces son reales" (pp. 22-24).

16 El propio Husserl (1995) sostiene que sin los artificios metódicos, es decir, amparados solo en la representación de contenidos objetivos, "nunca se les hubiera ocurrido a los hombres construir ciencias" (p. 43). 
ciencia utiliza métodos para ampliar tal grado de evidencia; de lo contrario, nunca se zafaría de los meros datos que están a la mano.

De acuerdo con lo anterior, no es difícil admitir en la concepción husserliana de ciencia la legitimidad en la postulación de entidades teóricas, esto por las siguientes razones: en primer lugar, si bien estas entidades no se dan al ojo desnudo, esto no implica que el científico las postule a modo de ficciones, sino que las respalda en evidencias que, aunque podrían tener al inicio un respaldo débil, a modo de hipótesis, posteriormente pueden ser comprobadas o rechazadas. En segundo lugar, el actuar del científico no está restringido a los objetos de tamaño medio; es claro que el limitarse a estos objetos no ha sido una característica de la ciencia, ni permitiría que esta ampliara su dominio de indagación; antes bien, Husserl muestra cómo el científico echa mano de métodos o instrumentos que, aunque respondan a la teoría de la esfera particular, permiten proponer nuevos juicios que encontrarán o no su cumplimiento en la experiencia. Finalmente, el conocimiento que se adquiere de estas entidades no se da de una vez por todas, no tanto por la precariedad del científico o la ciencia, sino por la misma condición de la cosa física (esta condición incluye tanto a las cosas físicas perceptibles a ojo desnudo como a los inobservables); esto no es óbice, sin embargo, para que pueda hablarse de EP de los juicios: puede ser que las entidades teóricas no gocen de un alto grado de EP; sin embargo, EP puede ir alcanzando grados más elevados o bien por el uso de nuevos y mejores instrumentos, o bien por la precisión de sus mediciones, o bien, por qué no, por el éxito de sus explicaciones. Esto último implica no una actitud agnóstica (escéptica) con respecto a las entidades teóricas, sino, al contrario, un compromiso con su existencia, la cual se va revelando de modos más precisos con el avance de la investigación científica.

Lo que a estas alturas debe quedar claro es que, a menos que se interprete PP desde el contexto empirista (como sospecho que lo hace Wiltsche, así sea desde el Empirismo Constructivo), interpretación por demás ajena a las pretensiones de Husserl, no habría respaldo suficiente para, en el terreno de la cosa física, postular tajantemente desde Husserl una distinción entre entidades observables y entidades teóricas.

\section{Conclusión}

La consideración del texto de Wiltsche permitió reflexionar acerca de la relevancia que puede tener Husserl en las discusiones contemporáneas sobre la ciencia; sin embargo, también permitió darnos cuenta de que en aras de tal relevancia no pueden sacrificarse tesis inherentes al programa fenomenológico. 
Si bien el artículo no ha planteado con suficiencia el tipo de realismo que adoptaría Husserl (pretensión que excede el propósito de este texto) -que en cualquier caso tendrá que estar alejado del Realismo metafísico ${ }^{17}$ y dependerá de una profunda investigación sobre el Idealismo trascendental de Husserl-al menos ha tratado de desligarlo del contexto antirrealista en el que pretendía adscribirlo Wiltsche; de igual forma, ha mostrado las complejidades que encierra considerar la fenomenología en el ámbito de la ciencia natural, más específicamente, la exploración fenomenológica del "origen constitutivo del mundo objetivo" y la "ontología a priori del mundo real" (Husserl, 1979, $\S 59)$, tareas necesarias e ineludibles de la fenomenología del presente.

\section{Bibliografía}

AGUIRRE, J. "Husserl y la filosofía de la ciencia: desafíos y posibilidades". In: J. Aguirre, L. Jaramillo. Cuadernos de epistemología, número 5: reflexiones en torno a la filosofía de la ciencia y la metodología. Popayán: Universidad del Cauca, 2011. Cap. 5, pp. 103-129.

BELOUSEK, D. "Husserl on Scientific Method and Conceptual Change: A realist Appraisal”. Synthese, Vol. 115, pp. 71-98, 1998.

BERNET, R. "Husserl's Transcendental Idealism Revisited". The New Yearbook for Phenomenology and Phenomenological Philosophy, Vol. IV, pp. 1-20, 2004.

CARTWRIGHT, N. "How the Laws of Physics Lie". Oxford: Oxford University Press, 1983.

DRUMMNOD, J. "Realism Versus Anti-Realism: A Husserlian Contribution". In: Sokolowski, R. Edmund Husserl and the Phenomenological Tradition. Washington: Catholic University of America Press, 1988. Cap. 5, pp. 87-106.

"Husserlian Intentionality and Non-Foundational Realism".

Dordrecht: Kluwer Academic Publishers, 1990.

GURWITSCH, A. "Phenomenology and the Theory of Science". Evanston: Northwestern University Press, 1974.

GUTTING, G. "Husserl and Scientific Realism". Philosophy and Phenomenological Research, Vol. 39, pp. 42-56, 1978.

HACKING, I. "Representing and Intervening. Introductory Topics in the Philosophy of Natural Science”. Cambridge: Cambridge University Press, 1983.

17 Mohanty (2011) ha caracterizado suficientemente el tipo de realismo al que confronta Husserl (curiosamente lo hace aludiendo al $\$ 52$ de "Ideas I"). Esta caracterización está en consonancia con la crítica al realismo metafísico hecha por Putnam (1987), al cual llamó: "Realismo con $R$ mayúscula". Infortunadamente, da la impresión de que Mohanty ubica a Husserl por fuera del marco del realismo científico, aunque la perspicaz exposición que realiza no hace sino confirmar un realismo de nuevo orden. 
HINTIKKA, J. "The notion of intuition in Husserl". Revue Internationale de Philosophie, Vol. 224, pp. 169-191, 2003.

HUSSERL, E. "Jahrbuch für Philosophie und phänomenologische Forschung. Erster Band. Teil I". Halle: Verlag von Max Niemeyer, 1913.

"Ideas relativas a una fenomenología pura y una filosofía fenomenológica". México: Fondo de Cultura Económica, 1962. "Meditaciones cartesianas". Madrid: Ediciones Paulinas, 1979.

. "Ideas pertaining to a pure phenomenology and to a phenomenological philosophy. First Book: General Introduction to a pure phenomenology". The Hague: Martinus Nijhoff Publishers, 1983.

. "Investigaciones lógicas". Madrid: Altaya, 1995.

. "La idea de la fenomenología". Barcelona: Herder, 2011.

KOCHAN, J. "Husserl and the Phenomenology of Science". Studies in History and Philosophy of Science, Vol. 42, pp. 467-471, 2011.

KOCHAN, J., SMITH, H. "Philosophy of Science". In: S. Luft, S. Overgaard. The Routledge Companion to Phenomenology. London: Routledge, 2011. Cap. 42, pp. 467-471.

LATOUR, B., WOOLGAR, S. "Laboratory Life: The Construction of Scientific Facts". Princeton: Princeton University Press, 1986.

MOHANTY, J. "Edmund Husserl's The Freiberg Years: 1916-1938". New Haven: Yale University Press, 2011.

MORAN, D., COHEN, J. “The Husserl Dictionary”. London: Continuum, 2010.

MORMANN, T. "Husserl's Philosophy of Science and the Semantic Approach". Philosophy of Science, Vol. 58, Nr. 1, pp. 3-27, 1991.

PALETTE, V. "Wahrheit". In: H. Gander. Husserl-Lexikon. Darmstadt: Wissenschaftliche Buchgesellschaft, 2010. pp. 304-306.

PUTNAM, H. "The many faces of Realism". LaSalle: Open Court Publishing Company, 1987.

ROUSE, J. "Husserlian Phenomenology and Scientific Realism". Philosophy of Science, Vol. 54, pp. 222-232, 1987.

SANKEY, H. "Scientific Realism and the Rationality of Science". Aldershot: Ashgate, 2008.

SAN MARTÍN, J. “La estructura del método fenomenológico". Madrid: UNED, 1986. SERRA, A. M. "Deckung". In: H. Gander. Husserl-Lexikon. Darmstadt: Wissenschaftliche Buchgesellschaft, 2010. pp. 55-57.

SOFFER, G. "Phenomenology and Scientific Realism: Husserl's critique of Galileo". Review of Metaphysics, Vol. 44, pp. 67-94, 1990.

STRÖKER, E. "The Husserlian Foundations of Science". Dordrecht: Kluwer Academic Publishers, 1997.

VALLOR, S. "The Pregnancy of the real: A phenomenological defense of experimental realism". Inquiry, Vol. 52, Nr. 2, pp. 1-25, 2009.

VAN FRAASSEN, B. “The Scientific Image”. Oxford: Clarendon Press, 1980. 
VAN FRAASSEN, B. “Against Transcendental Empiricism”. In: T. Staplenton. J. The Question of Hermeneutics. Dordrecht: Kluwer, 1994. pp. 309-335.

151, pp. 151-170, 2001.

"Constructive Empiricism Now". Philosophical Studies, Vol. . "The Empirical Stance”. New Haven: Yale University Press, 2002. . "From a View of Science to a New Empiricism". In: B. Monton. Images of Empiricism. Oxford: Oxford University Press, 2007. pp. 337-383.

WILTSCHE, H. "What is Wrong with Husserl's Scientific Anti-Realism?" Inquiry, Vol. 55, Nr. 2, pp. 105-130, 2012. 